Y. A. Rozanov

Nagoya Math. J.

Vol. 47 (1972), 227-235

\title{
ON NON-ANTICIPATIVE LINEAR TRANSFORMATIONS OF GAUSSIAN PROCESSES WITH EQUIVALENT DISTRIBUTIONS
}

\author{
YU. A. ROZANOV
}

Let $\xi(t), t \in T$, be a Gaussian process on a set $T$, and $H=H(\xi)$ be the closed linear manifold generated by all values $\xi(t), t \in T$, with the inner product

$$
\left\langle\eta_{1}, \eta_{2}\right\rangle=E \eta_{1} \eta_{2} ; \quad \eta_{1}, \eta_{2} \in H
$$

We suppose that the Hilbert space $H$ is separable.

Let $\mathscr{A}$ be a linear operator on $H$; we call a random process of the form

$$
\eta(t)=\mathscr{A} \xi(t), \quad t \in T,
$$

a linear transformation of the process $\xi(t), t \in T$. One says that a linear transformation $\mathscr{A}$ is non-anticipative, if

$$
\mathscr{A} H_{t}(\xi) \subseteq H_{t}(\xi), \quad t \in T,
$$

where $H_{t}(\xi)$ denotes the subspace in $H$, which is generated by all values $\xi(s), s \leq t$.

Let $P$ be a probability distribution of the Gaussian process $\xi=\xi(t)$, $t \in T$, in some measurable space $(X, \mathfrak{B}, P)$ of $\langle$ trajectories $\rangle x=x(t), t \in T$, where $\sigma$-algebra $\mathfrak{B}$ is generated by all sets $\{x(t) \in B\}(t \in T), B$ are Borel sets on the real line, so $P$ is determined by finite-dimensional distributions of the random process $\xi=\xi(t), t \in T$. Let $Q$ be a probability distribution of the Gaussian process $\eta=\eta(t), t \in T$, represented by the formula (1). According to well known Feldman's theorem (see, for example, [1]), $Q$ is equivalent to $P(Q \sim P)$ if and only if the operator

$$
\boldsymbol{B}=\mathscr{A}^{*} \mathscr{A}
$$

is invertible and $I-B \in S_{2}$, where $S_{2}$ denotes the class of all HilbertSchmidt operators in $H$.

Received January 18, 1972. 
The operator $\boldsymbol{B}$ connects with the correlation function $\boldsymbol{B}(s, t)$ of the Gaussian distribution $Q$ as

$$
\boldsymbol{B}(s, t)=\langle\boldsymbol{B} \xi(s), \xi(t)\rangle, \quad s, t \in T ;
$$

let us call $\boldsymbol{B}$ the correlation operator of $Q$. Obviously, for any equivalent distribution $Q$ (i.e. $Q$ has strictly positive correlation operator $\boldsymbol{B}$, such that $I \quad B \in S_{2}$.) there is a linear transformation (1), which gives us a Gaussian process $\eta(t), t \in T$, with the distribution $Q$ : the general operator $\mathscr{A}$, which satisfies the condition (3), has the form

$$
\mathscr{A}=V \boldsymbol{B}^{1 / 2}
$$

where $V$ is an arbitrary unitary operator in $H$.

Let us consider a linear transformation (1) with $\mathscr{A}=I-\Delta$ :

$$
\eta(t)=\xi(t)-\Delta \xi(t), \quad t \in T .
$$

It is more convenient to reformulate Feldman's theorem in the following way: $Q \sim P$ if and only if $I-B^{1 / 2} \in S_{2}$ and 1 does not belong to the spectrum of $I-\boldsymbol{B}^{1 / 2}$. Indeed, $I-\boldsymbol{B} \in S_{2}$ if and only if

$$
\left(I-B^{1 / 2}\right)=(I-B)\left(I+B^{1 / 2}\right)^{-1} \in S_{2} .
$$

It is easy to see that for any operator $\Delta \in S_{2}$, which has no eigenvalue equal to 1 , the random process $\eta(t), t \in T$, of the form (6) has the equivalent distribution $Q$ with the correlation operator, because

$$
I-B=\Delta+\Delta^{*}(I-\Delta) \in S_{2} .
$$

But the condition $\Delta \in S_{2}$ is not nesessary for the equivalence $Q \sim P$. Namely, by the formula (5) we have

$$
\Delta=I-V B^{1 / 2},
$$

where $V$ is some unitary operator and (for the equivalent distribution Q) $I-B^{1 / 2} \in S_{2} ;$ obviously $\Delta \in S_{2}$ if and only if $\left[\Delta-\left(I-B^{1 / 2}\right)\right] B^{-1 / 2}=$ $I-V \in S_{2}$.

Then we shall be interested in the linear transformation (6) with operators $\Delta \in S_{2}$. As we have obtained, it holds true if and only if

$$
I-V \in S_{2}
$$

where $V$ is an unitary operator connected with the operator $\Delta$ by the formula (7) : $\Delta=I-V \boldsymbol{B}^{1 / 2}$. According to Feldman's theorem any trans- 
formation (6) such that $\Delta \in S_{2}$ and 1 does not belong to the spectrum $\Delta$ gives a random process $\eta(t), t \in T$, with an equivalent distribution $Q$.

We shall be interested also in a such property of the linear transformation (6) as to be non-anticipative that means

$$
\Delta H_{t}(\xi) \subseteq H_{t}(\xi), \quad t \in T .
$$

In the resent time it was paid attention for non-anticipative transformations in connection with Hitsuda's result [2] for the Wiener process $\xi(t), 0 \leq t \leq 1$ : any Gaussian process $\eta(t), 0 \leq t \leq 1$, with an equivalent probability distribution can be represent in the form

$$
\eta(t)=\xi(t)-\int_{0}^{t}\left[\int_{0}^{s} \Delta(u, s) d \xi(u)\right] d s
$$

where $\Delta(t, s) ; 0 \leq t, s \leq 1$,

$$
\begin{aligned}
& \Delta(t, s)=0, \quad s<t, \\
& \int_{0}^{1} \int_{0}^{1} \Delta(t, s)^{2} d t d s<\infty .
\end{aligned}
$$

Though in the paper [2] it was used some theorems on the martingales, it was clear that the representation (10) can be obtained as a result of the theory of operators in a Hilbert space: the formula (10) is given by a non-anticipative transformation (6) with $\Delta \in S_{2}$ in the case of Wiener process $\xi(t), 0 \leq t \leq 1$. The existense of such transformation in the general case follows from non-trivial Gohberg-Krein's theorems on so-called special factorization; namely, any positive operator $\boldsymbol{B}$ of the type

$$
\begin{gathered}
B=(I-F)=(I-G)^{-1} \\
\left(F \text { and } G=-F B^{-1} \text { belong } S_{2}\right)
\end{gathered}
$$

can be represented in the form

$$
B=(I+X) \mathscr{D}\left(I+X^{*}\right)
$$

where $(I+X)$ is invertible, $X \in S_{2}$ and $\mathscr{D} \geq 0$; besides the operators $X$ and $\mathscr{D}$ satisfy the condition

$$
X H_{t} \subseteq H_{t}, \quad \mathscr{D} H_{t} \subseteq H_{t} \quad(t \in T)
$$

for a given monotone family of subspaces $H_{t}, t \in T\left(H_{s} \subseteq H_{t}\right.$ if $\left.s \leq t\right)$ (see the theorems 6.2 Ch. IV and 10.1 Ch. I in the book [3]). It is clear that for $H_{t}=H_{t}(\xi), t \in T$, the operator 


$$
\mathscr{A}=(I+X) \mathscr{D}^{1 / 2}
$$

satisfies the conditions (2) and (3), so the corresponding linear transformation (6) with $\Delta=I-\mathscr{A}$ will be non-anticipative. This proof of the existense of non-anticipative representations (6) for Gaussian processes $\eta(t), t \in T$, with equivalent distributions was suggested resently by Kallianpur and Oodaira [4] (in the case of Wiener process $\xi(t), 0 \leq t \leq 1$, it was done ealier by Kailath [5]). We should like to do the following essential note: for the operator $\mathscr{A}$, which was mentioned above (see (14)) it holds true that

$$
\Delta=I-\mathscr{A} \in S_{2},
$$

so for any Gaussian process $\xi(t), t \in T$, there is a non-anticipative Gaussian process $\eta(t)=\xi(t)-\Delta \xi(t), t \in T$ (where $\Delta \in S_{2}$ satisfies the condition (9)) with a given equivalent probability distribution.

Indeed, in the representation (13) we have $(I+X)^{-1}=I+\mathscr{T}, \mathscr{T}=$ $-X(I+X)^{-1} \in S_{2}$, and the operator $\mathscr{D}$ has a form

$$
\mathscr{D}=(I+\mathscr{T})(I-F)\left(I+\mathscr{T}^{*}\right)=I+V
$$

where

$$
V=\mathscr{T}\left(I-F^{\prime}\right)\left(I+\mathscr{T}^{*}\right)-F\left(I+\mathscr{T}^{*}\right)+\mathscr{T}^{*} \in S_{2} .
$$

From relations

$$
\begin{gathered}
\mathscr{D}^{1 / 2}=(I+V)^{1 / 2}=I+W, \\
I+V=(I+W)^{2}=I+W(2 I+W)=I+W\left(I+\mathscr{D}^{1 / 2}\right),
\end{gathered}
$$

we obtain that

$$
W=V\left(I+\mathscr{D}^{1 / 2}\right)^{-1} \in S_{2}
$$

so

$$
\begin{aligned}
\Delta & =I-\mathscr{A}=I-(I+X) \mathscr{D}^{1 / 2} \\
& =I-(I+X)(I+W)=-X(I+W)-W \in S_{2} .
\end{aligned}
$$

It is worth to pay attention for the following fact: the linear transformation (6) with the operator $\mathscr{A}=I-\Delta$ of the form (14) is such that

$$
H_{t}(\xi)=H_{t}(\eta), \quad t \in T
$$


Indeed, for the invertible positive operator $\mathscr{D}^{1 / 2}: \mathscr{D}^{1 / 2} H_{t}(\xi) \subseteq H_{t}(\xi)$, we have

$$
\mathscr{D}^{1 / 2} H_{t}(\xi)=H_{t}(\xi)
$$

because in a contrary case there is an element $h \in H_{t}(\xi)$, such that $h \perp \mathscr{D}^{1 / 2} H_{t}(\xi)$ and $\mathscr{D}^{1 / 2} h=0$. Remind that a Volterra operator $X$ has only one point of a spectra equal to 0 , so for the operator $(I+X)$ in the formula (14), $(I+X) H_{t}(\xi) \subseteq H_{t}(\xi)$, we have

$$
(I+X) H_{t}(\xi)=H_{t}(\xi) .
$$

Now it is obvious that the operator $\mathscr{A}=(I+X) \mathscr{D}^{1 / 2}$ satisfies the condition (16).

Let us consider a few examples of non-anticipative representations (6) with $\Delta \in S_{2}$.

EXAMPLE 1. Let $\xi(t), 0 \leq t \leq 1$, be a Gaussian process with stationary increments :

$$
\xi(t)=\int_{-\infty}^{\infty} \frac{e^{i \lambda t}-1}{i \lambda} \Phi(d \lambda),
$$

which has a spectral density $f(\lambda)$ of the type:

$$
0<\lim _{\lambda \rightarrow \infty} f(\lambda) \leq \varlimsup_{\lambda \rightarrow \infty} f(\lambda)<\infty
$$

(if $f(\lambda)=1 / 2 \pi$, we deal with Wiener process $\xi(t), 0 \leq t \leq 1$ ).

The corresponding space $H$ consists of all random variables

$$
\begin{gathered}
\eta=\int_{-\infty}^{\infty} \varphi(\lambda) \Phi(d \lambda)=\int_{0}^{1} c(t) \dot{\xi}(t) d t \\
\left(\varphi(\lambda)=\int_{0}^{1} e^{i \lambda t} c(t) d t\right)
\end{gathered}
$$

where functions $c(t), 0 \leq t \leq 1$, belonging to $L^{2}[0,1]$ and $\dot{\xi}(t)$ is the generalized delivative of process $\xi(t)$; besides $^{1)}$

$$
\|\eta\|^{2}=\int_{-\infty}^{\infty}|\varphi(\lambda)|^{2} d \lambda \bigcup \int_{0}^{1} \boldsymbol{c}(t)^{2} d t
$$

1) The relation $\alpha{ }_{n}^{\cup} \beta$ between variables $\alpha, \beta$ means that

$$
0<c_{1} \leq \frac{\alpha}{\beta} \leq c_{2}<\infty .
$$


(see, for example, [1] or [6]), and the formula (18) gives us the isomorphism between $H$ and $L^{2}[0,1]$ such that

$$
H_{t}(\xi) \leftrightarrow L^{2}[0, t], \quad 0 \leq t \leq 1,
$$

where $L^{2}[0, t]$ denotes the subspace of all functions $\boldsymbol{c}(s), 0 \leq s \leq 1: \boldsymbol{c}(s)$ $=0$ for $s>t$. As it follows from the conditions (19) and (20), the formula

$$
\Delta \eta=\int_{0}^{1}[\tilde{\Delta} c(t)] \dot{\xi}(t) d t
$$

gives us the isomorphism $\Delta \leftrightarrow \tilde{\Delta}$ between Hilbert-Schmidt operators in $H$ and $L^{2}[0,1]$, and an operator $\Delta$ satisfies the condition (9) if and only if

$$
\tilde{\Delta} L^{2}[0, t] \subseteq L^{2}[0, t], \quad 0 \leq t \leq 1,
$$

that is equivalent to the condition (11) for a corresponding kernel $\Delta(t, s)$ :

$$
\tilde{\Delta} \boldsymbol{c}(t)=\int_{0}^{1} \Delta(t, s) \boldsymbol{c}(s) d s, \quad 0 \leq t \leq 1,
$$

(remind $\tilde{\Delta} \in S_{2}$ if and only if $\Delta(t, s) ; 0 \leq t, s \leq 1$, satisfies the condition (12)). Thus any non-anticipative operator $\Delta \in S_{2}$ can be discribed by the formula

$$
\Delta \eta=\int_{0}^{1}\left[\int_{t}^{1} \Delta(t, s) c(s) d s\right] \dot{\xi}(t) d t
$$

with a Volterra, Hilbert-Schmidt kernel $\Delta(t, s) ; 0 \leq t \leq 1$. For variables $\xi(t), 0 \leq t \leq 1$, which correspond to the functions

$$
c(s)=\left\{\begin{array}{lc}
1, & 0 \leq s \leq t, \\
0, & s>t,
\end{array}\right.
$$

we obtained from the formula (21) a general non-anticipative transformation (6) with $\Delta \in S_{2}$ as

$$
\eta(t)=\xi(t)+\int_{0}^{t}\left[\int_{0}^{s} \Delta(u, s) \dot{\xi}(u) d u\right] d s, \quad 0 \leq t \leq 1,
$$

that gives us Hitsuda's representation (10) in the case of Wiener process $\xi(t), 0 \leq t \leq 1$.

ExAmple 2. Let $\xi(t), 0 \leq t \leq 1$, be a Gaussian stationary process: 


$$
\xi(t)=\int_{-\infty}^{\infty} e^{i \lambda t} \Phi(d \lambda)
$$

with a. spectral density $f(\lambda)$ of the type

$$
0<\lim _{\lambda \rightarrow \infty} \lambda^{2 n} f(\lambda) \leq \varlimsup_{\lambda \rightarrow \infty} \lambda^{2 n} f(\lambda)<\infty .
$$

It will be convenient to introduce the process

$$
\begin{aligned}
\zeta(t) & =\sum_{k=0}^{n-1}{ }_{n} C_{k+1}\left[\xi^{(k)}(t)-\xi^{(k)}(0)\right]+\int_{0}^{t} \xi(s) d s \\
& =\int_{-\infty}^{\infty} \frac{e^{i \lambda t}-1}{i \lambda}(1+i \lambda)^{n} \Phi(d \lambda), \quad 0 \leq t \leq 1 .
\end{aligned}
$$

Obviously, the spectral density of this process $\zeta(t)$ with stationary increments satisfies the condition (17) and we can use results of our example 1 for the process $\zeta(t), 0 \leq t \leq 1$.

As is known (see, for example, [1] or [6]) the Hilbert space $H=H(\xi)$ consists of all variables

$$
\begin{gathered}
\eta=\int_{-\infty}^{\infty} \varphi(\lambda) \Phi(d \lambda) \\
\left(\varphi(\lambda)=\sum_{k=0}^{n-1} c_{k}(i \lambda)^{k}+(1+i \lambda)^{n} \int_{0}^{1} e^{i \lambda t} c(t) d t\right)
\end{gathered}
$$

where $c_{1}, \cdots, c_{n-1}$ are arbitrary constants and $c(t), \in L^{2}[0,1]$ or

$$
\eta=\sum_{k=0}^{n-1} c_{k} \xi^{(k)}(0)+\int_{0}^{1} c(t) \dot{\zeta}(t) d t
$$

where $\dot{\zeta}(t)$ denotes the generalized derivative of the process $\zeta(t)$ determined by the transformation (24).

If we consider in the general formula (25) only functions $c(s)$, $\in L^{2}[0, t]$, we obtain the corresponding subspace $H_{t}(\xi), 0 \leq t \leq 1$, and it shows that $H_{t}(\xi)$ is a direct sum of the subspace

$$
H_{0+}(\xi)=\bigcap_{t>0} H_{t}(\xi),
$$

which consists of all variables $\eta=\sum_{k=0}^{n-1} c_{k} \xi^{(k)}(0)$, and the subspace $H_{t}(\zeta)$ of all variables $\eta=\int_{0}^{t} c(s) \dot{\zeta}(s) d s$ :

$$
H_{t}(\xi)=H_{0+}(\xi)+H_{t}(\zeta), \quad 0 \leq t \leq 1 ;
$$


in particular

$$
H(\xi)=H_{0+}(\xi)+H(\zeta)
$$

Let $P$ be a projector on the subspace $H(\zeta)$ parallel to the subspace $H_{0+}(\xi)$. If $\Delta \in S_{2}$ then $P \Delta P \in S_{2}$; obviously, if $\Delta$ satisfies the condition (9) then $P \triangle P$ satisfies to the similar condition with respect to $H_{t}(\zeta)$, $0 \leq t \leq 1$. As it has been shown (see (21)), the non-anticipative operator $P \Delta P$ in $H(\zeta)$ can be discribed by a Volterra, Hilbert-Schmidt kernel $\Delta(t, s) ; 0 \leq t, s \leq 1$ :

$$
P \Delta P_{\eta}=\int_{0}^{1}\left[\int_{t}^{1} \Delta(t, s) \boldsymbol{c}(s) d s\right] \dot{\zeta}(t) d t
$$

where $\eta \in H$ is given by the formula (25) and

$$
P_{\eta}=\int_{0}^{1} \boldsymbol{c}(t) \dot{\zeta}(t) d t
$$

For any non-anticipative operator $\Delta$ in $H(\xi)$ we have

$$
\Delta H_{0+}=\Delta\left(\bigcap_{t>0} H_{t}\right) \subseteq \bigcap_{t}\left(\Delta H_{t}\right) \subseteq \bigcap_{t} H_{t}=H_{0+}
$$

that is equivalent to the condition

$$
(I-P) \Delta(I-P)=\Delta(I-P) \text {. }
$$

Then

$$
\Delta=(I-P) \Delta P+\Delta(I-P)+P \Delta P=(I-P) \Delta+P \Delta P
$$

where the finite-dimensional operator $(I-P) \Delta$, mapping $H(\xi)$ on the subspace $H_{0+}(\xi)$, has the form

$$
(I-P) \Delta \eta=\sum_{k=0}^{n-1}\left\langle\eta, \eta_{k}\right\rangle \xi^{(k)}(0)
$$

$\left(\eta_{0}, \eta_{1}, \cdots, \eta_{n-1}\right.$ are some fixed elements in $\left.H_{0+}\right)$. Combining formulas (26) and (27), we obtain a general non-anticipative operator $\Delta \in S$ as

$$
\Delta \eta=\sum_{k=0}^{n-1}\left\langle\eta, \eta_{k}\right\rangle \xi^{(k)}(0)+\int_{0}^{1}\left[\int_{t}^{1} \Delta(t, s) c(s) d s\right] \dot{\zeta}(t) d t
$$

in particular, for $\eta \in H_{t}(\xi)$

$$
\Delta \eta=\sum_{k=0}^{n-1}\left\langle\eta, \eta_{k}\right\rangle \xi^{(k)}(0)+\int_{0}^{t}\left[\int_{0}^{s} \Delta(u, s) \dot{\zeta}(u) d u\right] c(s) d s .
$$




\section{REFERENCES}

[1] Rozanov, Yu A., Infinite-dimensional Gaussian distributions (in Russian), Proc. Steklov Math. Inst., 108, 1968 (English translation Amer. Math. Soc., Providence, 1971).

[2] Hitsuda, M., Representation of Gaussian processes equivalent to Wiener process, Osaka J. Math., 5, 299-312, 1968.

[ 3 ] Gohberg, I. C. and Krein, M. G., Theory and applications of Volterra operators in Hilbert space (in Russian), M., Nauka, 1967 (English translation: Amer. Math. Soc., Providence, 1970).

[ 4 ] Kallianpur, G. and Oodaira, H., Non-anticipative representations of equivalent Gaussian processes (in print).

[5] Kailath, T., Likelihood ratios of Gaussian processes, IEEE Trans. Information theory IT-16, 276-288, 1970.

[6 ] Ibragimov, I. A. and Rozanov, Yu. A., Gaussian random processes (in Russian), M., "Nauka", 1970.

Steklov Mathematical Institute, Moscow 